\title{
Physiotherapy Management of Copd Exacerbations
}

\author{
Keymer $\mathrm{J}^{\mathbf{1} 2 *}$, León $\mathbf{J}^{\mathbf{1}}$, Molina $\mathbf{J}^{\mathbf{2}}$ and Giugliano-Jaramillo $\mathrm{C}^{\mathbf{1}}$ \\ ${ }^{1}$ Servicio De Medicina Física Y Rehabilitación, Clínica Alemana De Santiago, \\ Santiago, Chile \\ ${ }^{2}$ Escuela De Kinesiología, Universidad Del Desarrollo, Santiago, Chile \\ *Corresponding Author: Keymer J, Servicio De Medicina Física Y Rehabilitación, \\ Clínica Alemana De Santiago, Santiago, Chile.
}

Received: January 02, 2020

Published: February 08, 2020

(C) All rights are reserved by Keymer J.

DOI: $10.31080 /$ ASMS.2020.04.0558

\begin{abstract}
Physiotherapy remains an important tool in the treatment of cronic obstructive pulmonary disease (COPD) and especially when this disease presents exacerbations that lead to hospitalize our patients. In this article we will analyze how the physiotherapist can help these patients through respiratory therapy using, non-invasive ventilation (NIV), positioning, aerosol therapy, humidification and assistance in fibroboncoscopy.

Keywords: Physiotherapy; COPD; COPD Exacerbations; NIV
\end{abstract}

\section{Introduction}

Airflow limitation is usually progressive and associated with an abnormal inflammatory response of the lungs to noxious particles or gases [1]. The global initiative for chronic obstructive lung disease is a global entity that published in 2001 a consensus and global strategy reports for the diagnosis and prevention of cronic obstructive pulmonary disease (COPD). The reports published in 2013, 2014, 2015 and 2017 were based on the scientific literature published since 2011, but maintain the same paradigm of treatment. Only in 2015 the Global Initiative for Chronic Obstructive Lung Disease (GOLD) and The Global Initiative for Asthma (GINA) approved the agreements prepared by a scientific committee for the management of Asthma and COPD overlap syndrome. Now we can see that COPD defintion until 2017 has been change progressively in the limitation of the air flow and that is associated to an unexpected inflammatory answer of lungs caused by gases or noxious particles.

COPD is a common, preventable and treatable disease that is characterized by persistent respiratory symptoms and airflow limitation that is caused by airway and/or alveolar abnormalities usually caused by signicant exposure to noxious particles or gases2. Since the definition of GOLD 2017, they acknowledge that it is an abnormal inflammatory response and define it as a frequent, prevenible and treatable disease. It is also characterized by persistent respiratory symptoms and by limitation of air flow, kept from the previous definition. Also defines that this limitation must result in anomalies in the respiratory and alveolar airways caused by exposure to particles and harmful agents. This definition is roughly similar but should clarify that COPD is associated with an inflammatory response, that is a thing of the past.
Exacerbations (definition, diagnosis and treatment) are extensively reviewed, recommendations are given for acute treatment and its prevention, and criteria for follow-up after discharge are given. Analizing deeper the GOLD report, it highlights the importance of symptomology during lung growth and development. This report of 2017 exhaustively checks COPD exacerbations, giving a definition to them, diagnosis and recommendations for their treatment, prevention and criteria for after-following up of patients. Pharmacological and non-pharmacological therapies are revalued and updated. It includes sections for the treatment of emphysema, self-management, pulmonary rehabilitation, revision of the inhalation technique, oxygen therapy and non-invasive mechanical ventilation. Here it distinguishes the importance of pulmonary rehabilitation through physical therapy, the aditional oxygen therapy must be used during exercise and the importance of a good education for the inhalation technique. The importance of examining symptoms and the risk of future exacerbations for the treatment of stable COPD is emphasized; Changes to a more personalized approach to treatment with pharmacotherapeutic treatment strategies (step up and down) are included. The importance of non-invasive ventilation (NIV), what is a main theme to develop, and the improvement of pharmacological treatment in COPD exacerbations. In fact, one of the changes that they proposed is the early treatment with two long action broncodilators now you can ask what is the best way of aerosol administration, which we will discuss later.

\section{Epidemiology}

In the United States, COPD affects men more than women, almost doubleing it, $4-6 \%$ of mens and $1-3 \%$ of women. The prevalence of COPD is two million, the second cause of invalidity and the fourth cause of death. This prevalence and mortality has increased in the last 25 years. In Chile COPD causes ten percent of hospitaliza- 
tions in adults. Is also the $9^{\text {th }}$ cause of death in our country, which means between four to five daily deaths. Santiago, the capital of Chile, is a city with great environmental pollution, that increases in winter affecting $9 \%$ of the population. The prevalence is greater in senior population, males over 40 years, smokers and exhibitors. The costs involving public health of each country is important and the European Union has estimated that a $6 \%$ of the total budget in health is spent by respiratory diseases and $56 \%$ of this $6 \%$ is spent in COPD, which corresponds (by 2014 ERS and ELF data) to approximately 380 billion euros. In 2004, COPD was the $13^{\text {nth }}$ morbility cause discribed by the world classification and it is expected that by 2030 will be the $5^{\text {th }}$ cause of morbility at world level.

\section{Pathways to the diagnosis of COPD}

In COPD we can observe different symptoms and risk factors. Especially during the anamnesis we must ask about: respiratory difficulty, chronic cough, secretion increase, tobacco habbit, patient work type and environmental characteristic [2]. Spirometry is important to establish diagnosis, but it must be complemented with other exams, such us x-rays and computerized axial tomography. Spirometry in a normal patient has a forced vital capacity (FVC) of 5 liters and a forced expiratory volume $\left(\mathrm{FEV}_{1}\right)$ of 4 liters. This changes in a COPD patient, were his FVC is 3.2 liters and the $\mathrm{FEV}_{1}$ of 0.8 liters [3] (Figure 1).
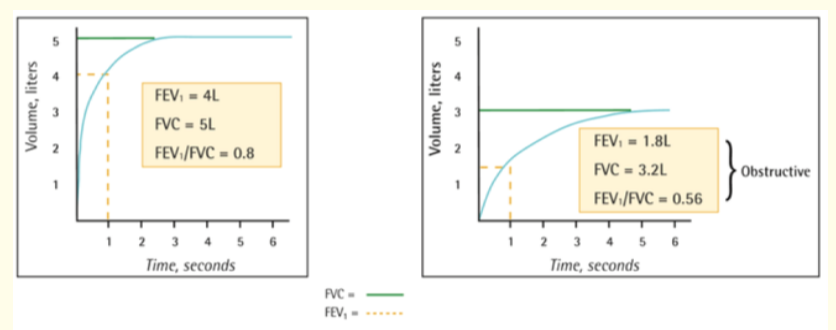

Figure 1: Left figure shows a normal spirometry. Patient has a FVC of 5 liters and a FEV1 of 4 liters. Right figure shows a COPD patient spirometry, were FVC is 3.2 liters and the FEV1 of 0.8 liters.

Global Initiative for Chronic Obstructive Lung Disease, 2017.

\section{Respiratory mechanical alteration}

As to the amendment of the respiratory mechanic of our patients, this point is of great importance to analyze and we, as physiotherapists, have the duty to know very well how the diaphragm works and what our treatment goal should be. these types of patients have an increase in functional residual capacity due the air entrapment. We should remember extremly well the diaphragm biomechanic, which is the main muscle of breathing. We know that patients with COPD have a different pattern of the diaphragm and therefore the biomechanic effectiveness of this muscle will be at disadvantage, given by the decrease of the aposition area. Therefore one of the most important objectives of physiotherapists should be giving a good point of support for the diaphragm to increase the appointment zone to improve muscle excursion. This can be made by manual therapy, giving a small resistance in the low cost zone (Figure2).

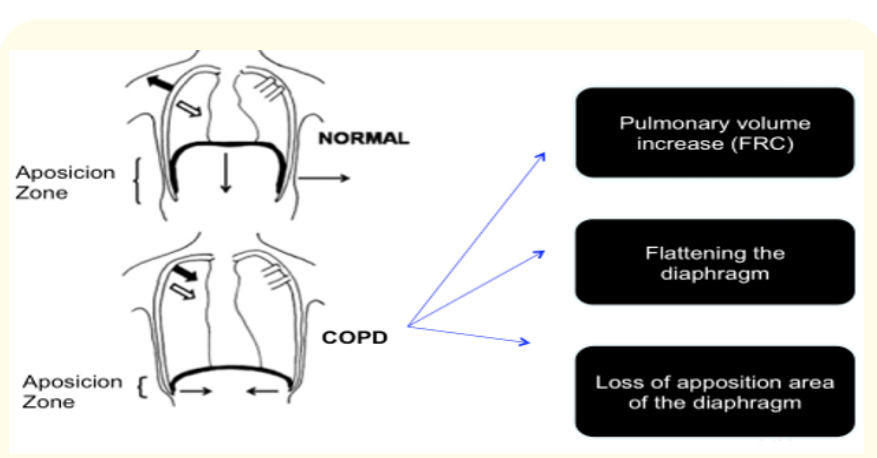

Figure 2: The picture above shows the normal apoosition zone of the Diaphragme muscle. The picture below shows the Diaphragme position in COPD patient, with the change in the apposition zone. Jimenez Manuel; Cervera Emilio; Libro Respiratorio: Prevención y rehabilitación en patología respiratoria crónica; Editorial Universidad Catolica de Mursia, 2018.

Not only we know that the pattern of the diaphragm is the responsible for air flow increase. We also know that patients have an increase of inflammation reaction and edema on small airway, mainly given by irritation. Similar to eye irritation when is in contact with smoke, that irritates and cries. This will produce increase in resistance and greater air traffic. COPD patients also have a decrease in mucociliary scaler movements, which leads to greater number of mucocellular plugs at bronchioles [4].

The mucocillary climber has a normal oscillatory cile movement called metacronal. In COPD the sun layer dehydrates and produce a decrease in ciliar movement [5]. This produces that normal physiological mechanisms fails and therefore diminishes the ability to eliminate secretions. Specialy COPD patients which produce more secrections because of cellular irritation and poor mucociliary scaler movement. This is why it is so important to maintain adecuate humidification when using NIV.

\section{Exacerbation of COPD}

In the last guide published by the European Respiratory Society with the American Thoracic Society in 2017, exacerbations are clinically defined as episodes of increased respiratory symptoms, particularly dyspnea, cough and sputum production, with increased purulence. COPD exacerbations (EA) have a negative impact in quality of life, accelerating disease progression, which may lead to frequent hospitalizations and death [6]. Definition has been kept similar, but was recently added the increase of purulence on secretions.

NIV therapy decreases intubation rate, mortality, eliminates the complications associated with therapy and decreases ICU and hospital lengh of stay in Patients with acute exacerbation of chronic respiratory insufficiency. Due to this reasons, NIV significantly diminishes economic expenses in care units and public health.

Osadnik, 2017 in a Cocrane systematic review identified 17 studies. This highlights that only few studies specified if the re- 
spiratory failure was hypoxemic or hypercapnic. In the studies it has been made a comparison between usual treatment versus NIV treatment. The results of the patients who received only NIV showed:

1. NIV decreases mortality risk in $46 \%$ (RR 0,54, ci $95 \% 038$ - 076).

2. As regards the likelihood of intubation, NIV decreases $65 \%$ (RR 036, 95\% CI 028 - 046).

3. Hospital stay also decrease in patients with NIV (MD -3.39days, 95\% CI-5.93 - 0.85).

4. Complication unrealted to NIV (RR0 26,95\%CI 013 - 053).

Therefore, the european society and the american society recommendations related NIV use in hospitalized patients with acute respiratory failure due to COPD is strong, A type evidence.

The etiology of COPD exacerbations, are the small airway increase inflammation, present in approximately $8 \%$ of viral or bacterial infections. A lower percentage is because environmental pollution or treatment breach.

It is important to mention that another symptom that has not beenformaly described in literatur is anguish wich makes more difficult to manage clinical symptoms. Patients with COPD exacerbation may be aggravated, even more, if you added co-morbidity as: pneumonia, bronchial cancer with atelectasias, cardiac insufficiency, pulmonary embolism or pneumothorax [7].

Airway damage is caused by the factors mentioned above, which may be aggravated by smoking habit. Cigarette smoke will damage the mucocilar scale and can enhance bacterial colonization, increasing mucus production, inflamation given by enzymes and cytokines that increases the elastic activity in the lung by producing an alteration in the balance of elastasa and anti elastasa [8] and perpetuating a vicious cicle.

Therefore, who experience frequent exacerbations of COPD have increased mortality, reduced exercise capacity, and muscle atrophy [9].

The clinical management of COPD exacerbations is broncodilators use.

It is known that broncodilators success depends on the application technique, because it is possible to lose up to $9 \%$ of the medicinal product. That is why we have to optimize the application and there are different devices: measured dose inhaler, dry powder, jet nebulizer and last the most efficient device is the mesh inhaler. The new GOLD 2017 establishes the treatment of broncoodilatation as the main pharmacological treatment and the evidence of support is based on diverse combinations of double broncoodilatation therapy. We must also apply corticosteroidal and antibiotic therapy for management.
Oxygenotherapy is also fundamental, because it doubles life expectancy of COPD patients, delaying its natural evolution up to two years, reduces disnea and miocardious work, which makes it an excellent complement for rehabilitation. NIV use also improves bronchial hygiene which is of great importance for respiratory therapy. A good respiratory therapy helps patients on the reduction of secretions and reliefs respiratory work.

Physiotherapy goal should be, to prevent and to treat complications of prolonged hospital length of stay and of COPD exacerbations. This is given due to reduce movement asociated to dysnoea fatigue and reduced functional capacity.

Here we have to intervene with specific respiratory objectives to maintain the permeable airway through bronchial drainage, cough assistance, huffins and suction when cough is weak. Improving the strength of respiratory muscules with exercises and devices such as treshold IMT valveTM. Improving ventilatory mechanic with seated position or at least forty-five degrees to reduce bronco aspiration risk and pneumonia [10]. Respiratory excercises are fundamental to prevent physical design. Today in numerous publications early mobilization is described supported by electro stimulation passive movement, to prevent deep venous thrombosis with antiembolic tights. The importance of passive mobilization from the first hours of hospitalization to avoid muscular breakdown, which are going to impair the rehabilitation.

Some of the treatment techniques suggested in the GOLD guide 2017 are the following:

1. Pulmonary rehabilitation improves disnea excersise tolerance in stable patients.

2. Oxygen therapy has a good level of evidence A, since its administration in the long term, increases survival in patients with severe chronic hypoxemy.

3. Patients with stable COPD and moderate functional capacity or exercise-induced arterial desaturation, long term oxygen prescription by itself does not decrease death rate, hospitalization or provide sustained benefits in health status, lung function and six minute walk distance. Moderate evidence level.

4. Traveling by airplane can increase hypoxemia level supported by low evidence (C)

5. Withing the ventilatory support, especifically the NIV, the GOLD review proposes that improves survival as domiciliary treatment in selected patients that come from recent hospitalization, particularly in patients with persistent hypercapnia with a $\mathrm{paCO}_{2}$ of $52 \mathrm{mmHg}$. This affirmation has an evidence level B.

The next point to treat is NIV treatment with an A evidence level. That is why NIV is recommended as a first ventilation mode to be used in patients with COPD with acute respiratory failure. NIV does not have contraindications and helps improving gas exchange and 
work of breath. NIV will also decrease hospitalization days and improve survival [2].

NIV indications according to the GOLD [2], our patients must present at least one of the following symptoms:

1. Respiratory acidosis. $\mathrm{PCO}_{2}$ shoul be greater than $45 \mathrm{mmHg}$ or $6 \mathrm{kPa}$. Ph should be lower than 7,35.

2. Disnea with clinical signs like respiratory muscule fatigue, with an increase of respiratory work. This can be observed when there is use of accessory musculature, paradox breathing or intercostal retraction. Therefore, it is highly important to observe our patients during the clinical exam.

3. Persistence of hypoxemia or desaturation despite using oxygen therapy.

Nevertheless, NIV is not the final solution in COPD treatment, that is why it is very important to reevaluated the patient after 1 - 2 hours, and if the patient does not improve, intubation and mechanical ventilation should be the next step [11].

Invasive mechanical ventilation indications are [12]:

1. Not tolerated or failed NIV after 1 - 2 hours.

2. Respiratory or cardiac arrest.

3. Decreased consciousness, psychomotor agitation or when sedation is not able to manage patients effort and dysnea.

4. Patients that present aspiration or vomiting, and airways protection is needed.

5. To help eliminate an abundant amount of secrections. This is controversial because it will depends on good respiratory therapy that can manage this.

6. Hemodynamic instability, management of water balance and vasoactive drugs.

7. Patients with serious ventricular or supra ventricular arritmia.

8. Treatment of serious hypoxemia in patients with $\mathrm{PaO}_{2} /$ $\mathrm{FiO}_{2}<146$ in which NIV should not be used.

Our group on 2015, in a review [13] describe that in COPD, NIV must be the first line treatment, giving a chance to avoid intubation, NIV have evidence 1A on hypercapnic failure, immunodepressed patients and pulmonary edema. Almost NIV have good evidence in IMV weaning of patients with COPD diagnosis.

NIV can also be used in other diagnosis such as pneumonia, distress and during broncoscopy procedure. In a prospective study our group developed in 2018 [14] in 28 patients, in need of diagnostic and/or therapeutic bronchoscopy in patients with some type of respiratory pathology. Of the 28 patients, 7 had COPD diagnosis and we founded that none of this COPD patients had IMV needs 24 hours after the procedure, there were no deaths and the average duration of the procedure was 7 minutes. With this results we could consider NIV as an alternative to avoid orotraqueal intubation in COPD patients that requires a bronchoscopy procedure, that may increase morbimortality in this group of patient.

\section{How the NIV helps COPD patients}

In a normal patient, the diaphragm contraction produces negative intrathoracic pressure, this generates an air flow from the outside to the alveoli. We know that inspiration is the result of muscles activity, but this does not happen in expiration. Expiration is a passive process that is given by elastic retraction of the thoracopulmonary system, generating the release of inspired air. The expiratory flow is enable until the airway pressure equals to atmosphere pressure. Where this occurs is called equal pressure point (EPP) that in a normal person is located in the pharynx, given to this a sufficient distance from the carina (Figure 3).

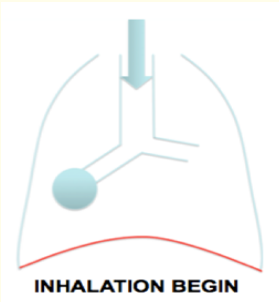

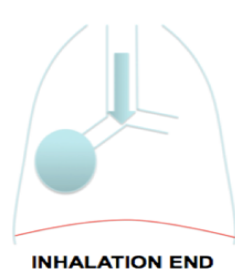

Figure 3: Keymer J.

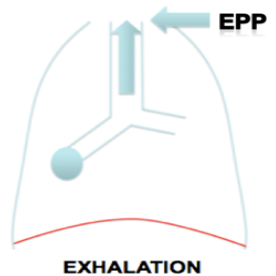

EXHALATION
COPD patiens lacks alfa 1 antitripsin enzyme in the peripherical airway walls, loosing its elastic properties wich makes its colaps. This is why COPD patiens have air trapment and CO2 retencion. While expiration is a passive proces, having their peripherial airway damaged, colaps puts resistance to airway flow avoiding its entirely releas, and equal pressure point is reach early in colapsable airway, closer to the carina (Figure 4).

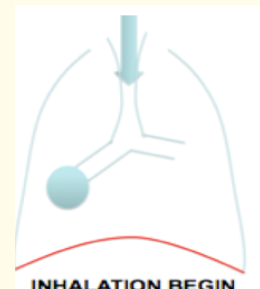

INHALATION BEGIN

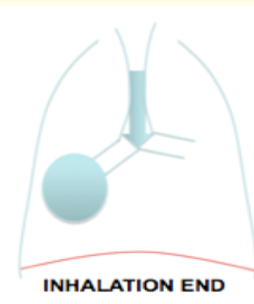

Figure 4: Keymer J.

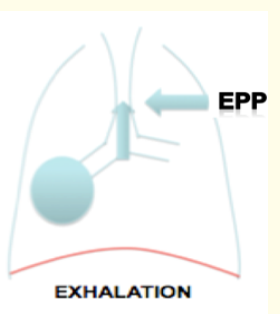

EXHALATION

(1)

In a COPD patient who has NIV, positive pressure stabilizes the central and peripheral airway collapse [15]. Air flows from outside to alveolis during inspiration by diaphragmatic contraction, and during expiration, that is passive, we do not have our peripheral and central support, and positive pressure decrease the airway resistance and allows lungs full deflated. The equal pressure point is restored near our pharynx, decreasing air intake and $\mathrm{CO}_{2}$ retention (Figure 5).

Respiratory management of the patient with acute respiratory failure

NIV indications in COPD exacerbations are:

1. Moderate to severe dyspnea. 
2. Tachypnea ( $24 \mathrm{bpm}$ in hypercapnia failure and $30 \mathrm{bpm}$ in hypoxemic failure).

3. Increase work of breathing with accessory mucles use.

4. $\mathrm{PaCO}_{2}>45 \mathrm{mmHg}$ in hypercapnia, and $50 \mathrm{mmHg}$ in hypoxemic patients.

5. $\mathrm{pH}<7.35$.

6. $\mathrm{PaO}_{2} / \mathrm{FiO}_{2}<200$.

NIV must be a bedside decision and need an exhaustive evaluation, where patient NIV tolerance, reduce work of breath and therapy efficacy should be considered.

Arterial gases examination should also be done. If patient does not improve their arterial gases in 1 - 2 hours and keeps its clinical signs, it should be intubated. If patient improves its arterial gases within the hour and decreases its clinical signs, we should continue with NIV therapy, always observing patients failure signs.

NIV weaning must be done when patients are clinically stable, tolerate NIV pressure decrease and tolerate hours without NIV during the day.

In another work that we published in the chilena journal of intensive medicine in 2010 [16], we try to prove that the NIV was able to avoid intubation in patients that entered in our ICU, where $14 \%$ of patients had COPD diagnosis. In this study $75 \%$ of the patients were rescued with NIV and only $25 \%$ failed and must be connect to invasive mechanical ventilation. Patients with acute respiratory failure that fail in the NIV present a significantly lower $\mathrm{PaO}_{2} / \mathrm{FiO}_{2}$ previous and during NIV therapy. Therefore, the initial $\mathrm{PaO}_{2} / \mathrm{FiO}_{2}$ and its not improve in the first 2 hours of NIV would be good predictors of need of intubation.

\section{Humidification and aerosolterapy in NIV}

Cerpa., et al. 2015, in an active humidification in NIV patients review [17], describe that clinic gases are dry, patients respiratory frequency should be normal or high, and NIV used is prolonged makes the arway dry. The NIV high unidirectional flow dries the upper airway and make that heat and moisture were lost. The lower diameter of the nasal airways induces an increase respistance in NIV inspiratory flow. This may affect the tolerance of NIV. All these affects ciliar activity, mocous secretion, decrease local blood flow and nasal air resistance, that finally may produce epithelium queratinization and mucocillary scaler effectiveness.

Therefore, the use of active overweight humidification in NIV is recommended, where fisher and paykel MR 810TM that is not servo-controlled is the most frecuently used.

It is recommended to install active humidification in patients with continouse need of NIV, for more than 24 hours, airway bleeding, hypothermia and patients that have amount or thick secrecions.
Aerosol therapy must be delivery in NIV patients, but is not an easy task. There is necessary to minimize aerosol losses through the mask, circuit and upper airway levels [18]. To optimize the drug delivery to the lungs, there are some devices that make this more eficent. Measured dose inhaler (MDI) with aerochamber, jet or mesh nebulizer, must go between the mask and the circuit exalatory port. The nebulization is very important and must go in combination with the NIV, the mask must not be removed when you need to nebulize a patient. Mesh nebulizer is the better option in NIV patients because it does not need additional air flow and deliver more homogeneous and lower drug size particles [19].

\section{Conclusion}

The physiotherapy is fundamental in the management of COPD patient, not only for respiratory and general exercises (not mentioned in this article. The physiotherapy has a fundamental rol in the management of NIV, since its instalation, setting, monitorization and weanning. Along with this, have to control the correct patient positioning, aerosolterapy, humidification and assistance in procedures like boncoscopy.

\section{Bibliography}

1. Global Initiative for Chronic Obstructive Lung Disease (2016).

2. Global Initiative for Chronic Obstructive Lung Disease (2017).

3. Casanova C., et al. "Prevalence of persistent blood eosinophilia: relation to outcomes in patients with COPD”. European Respiratory Journal 50.5 (2017).

4. Aaron SD., et al. "Granulocyte inflammatory markers and airway infection during acute exacerbation of chronic obstructive pulmonary disease". American Journal of Respiratory and Critical Care Medicine 163 (2001): 349-355.

5. Gheber L and Priel Z. "Extraction of cilium beat parameters by the combined application of photoelectric measurements and computer simulation". Biophysical Journal 72 (1997): 449-462.

6. Debray MP., et al. "Early computed tomography modifications following bronchial thermoplasty in patients with severe asthma". European Respiratory Journal 49.3 (2017).

7. Cruz E. “COPD Exacerbations: Definition and Prognostic Signification". Revista Chilena de Enfermedades Respiratorias 20.2 (2004): 76-79.

8. Murphy TF and Sethi S. "Bacterial infection in chronic obstructive pulmonary disease". The American Review of Respiratory Disease 146 (1992): 1067-1083.

9. Mikelsons C and Wedzicha W. "Pulmonary rehabilitation and the COPD exacerbation". Seminars in Respiratory and Critical Care Medicine 30.6 (2009): 649-655.

10. Muscedere J., et al. "The impact of frailty on intensive care unit outcomes: a systematic review and meta-analysis". Intensive Care Medicine 43.8 (2017): 1105-1122. 
11. Hill NS., et al. "Noninvasive ventilation in acute respiratory failure". Critical Care Medicine 35.10 (2007): 2402-2407.

12. Antonelli M., et al. "Predictors of failure of noninvasive positive pressure ventilation in patients with acute hypoxemic respiratory failure: a multi-center study". Intensive Care Medicine 27.11 (2001):1718-1728.

13. Hidalgo V., et al. "Noninvasive Mechanical Ventilation in Acute Respiratory Failure Patients: A Respiratory Therapist Perspective". The Open Respiratory Medicine Journal (2015): 120126.

14. Giugliano-Jaramillo C., et al. "Clinical and Breathing Behavior in Subjects Undergoing Bronchoscopy Supported with Noninvasive Mechanical Ventilation". Journal of Respiratory Medicine 2 (2018): 1 .

15. Ambrosino $\mathrm{N}$ and Xie L. "The Use of Non-invasive Ventilation during Exercise Training in COPD Patients". Chronic Obstructive Pulmonary Disease COPD 14.4 (2017): 396-400.

16. Hidalgo V., et al. "Evaluación de la ventilación mecánica no invasiva en paciente con insuficiencia respiratoria aguda". Revista Chilena de Medicina Intensiva25.1 (2010): 15-22.

17. Cerpa F., et al. "Humidification on Ventilated Patients: Heated Humidifications or Heat and Moisture Exchangers?" The Open Respiratory Medicine Journal 9 (2015): 104-111.

18. Dhand R. "Aerosol therapy in patients receiving noninvasive positive pressure ventilation". Journal of Aerosol Medicine and Pulmonary Drug Delivery 25.2 (2012): 63-79.

19. Keymer J., et al. "Inhalation Theraphy in Mechanical Ventilation". Mechanical Ventilation inthe ICU (2018).

\section{Assets from publication with us}

- Prompt Acknowledgement after receiving the article

- Thorough Double blinded peer review

- Rapid Publication

- Issue of Publication Certificate

- High visibility of your Published work

Website: https://www.actascientific.com/

Submit Article: https://www.actascientific.com/submission.php Email us: editor@actascientific.com

Contact us: +919182824667 